\title{
Penile prosthetic surgery for the management of Peyronie's disease
}

\author{
Omer A. Raheem, Tung-Chin Hsieh \\ Department of Urology, University of California San Diego Health, San Diego, California, USA \\ Contributions: (I) Conception and Design: All authors; (II) Administrative support: All authors; (III) Provision of study materials or patients: All \\ authors; (IV) Collection and assembly of data: All authors; (V) Data analysis and interpretation: All authors; (VI) Manuscript writing: All authors; (VII) \\ Final approval of manuscript: All authors. \\ Correspondence to: Tung-Chin Hsieh, MD. Department of Urology, UC San Diego Health, 200 West Arbor Drive \#8897, San Diego, CA 92103- \\ 8897, USA. Email: tmikehsieh@ucsd.edu.
}

\begin{abstract}
With the increased prevalence of Peyronie's disease (PD) among men, there is also an up-rise of associated erectile dysfunction (ED) which can be devastating psychologically and sexually to most men. A wide range of therapies including medical and surgical procedures are available to satisfactorily address this condition. However when ED coexists, the gold standard treatment is penile prosthetic prosthesis (PP) with or without additional straightening procedures. We herein systematically review the contemporary literature addressing the role of the PP in the management of PD with concomitant ED with particular emphasis on patient's satisfaction, outcomes and safety.
\end{abstract}

Keywords: Penile prosthesis; corporal fibrosis; erectile dysfunction (ED); Peyronie's disease (PD); therapeutic options

Submitted Feb 04, 2017. Accepted for publication Mar 21, 2017.

doi: $10.21037 /$ tau.2017.03.83

View this article at: http://dx.doi.org/10.21037/tau.2017.03.83

\section{Introduction}

Peyronie's disease (PD) is defined as a wound healing disorder of penile tunica albuginea leading to corporal fibrosis that can be psychologically devastating for affected men. The estimated prevalence of PD in the general male population ranges between $3 \%$ and $9 \%$, and is considerably higher than those reported historically (1). Although the underlying etiologic factors contributing for PD are not fully understood, idiopathic factors constitute up to $70 \%$. Additional risk factors for PD have postulated such as repetitive penile microtrauma leading to microvascular injury, buckling during sexual-related events, prior penile prosthesis (PP) implantation, prior infectionrelated PP explanation, priapism or prolonged use of an intracavernosal injection agent (1). PD is categorized into two pathologic phases: the early acute inflammatory phase and late chronic fibrotic phase. Men in the acute phase can present with penile pain upon erection and intercourse in $15-30 \%$ of cases, as well as progressive penile curvature. The pain is usually self limited and usually resolves within a year, whereas, the late chronic phase follows the resolution of inflammation and stabilization of penile deformity. It usually occurs within 12-18 months following initial insult (2). Moreover, PD patients often present with various challenging clinical features as a result of penile and/ or corporal fibrosis: palpable penile plaques, hourglass defects, penile hinging, and penile shortening (1). In addition to functional deformities, there is an association between PD and erectile dysfunction (ED) in approximately $30 \%$ of cases $(1,2)$. Affected patients often suffer from associated psychological distress, which may lead to strained interpersonal relationships and diminished quality of life (3). In essence, the pathophysiology of ED associated $\mathrm{PD}$ is usually related to penile discomfort, significant penile curvature, angulation and/or shortening limiting penetrative sexual function. The primary goals of surgical treatment are to correct the penile deformity, preserve or restore erectile function, and prevent loss of penile size. The choice of surgical procedure is based on the degree of penile curvature, penile length, presence of extensive plaque calcifications, patient's preoperative erectile function, 
prior failed medical therapy and patient expectations and desired long term outcome $(3,4)$. Both medical and surgical treatment options are available in the management of PD including PP, particularly in men with concomitant ED. In this systematic review, we sought to review the contemporary published literature addressing the role of the $\mathrm{PP}$ in the management of PD.

\section{Methods}

A detailed, comprehensive literature review was performed to identify all published peer-reviewed articles which PP and $\mathrm{PD}$ in the urological literature over a 16 -year period, i.e., between 2000 and 2016. The search was conducted through MEDLINE ${ }^{\circledR}$ database, the Cochrane Library ${ }^{\circledR}$ Central Search, Web of Science and Google Scholar. The initial search terms were PP and PD. Search results were screened for appropriate studies with particular emphasis placed on clinical and experimental studies as well as review articles. Articles referenced were screened to maximize review and inclusion of pertinent data. While English language text was not a specific search parameter, only English language publications were considered. All relevant studies collected were carefully examined to extract relevant data pertained to PP and PD.

\section{Evidence synthesis for this systematic review}

\section{Therapeutic options for PD: medical therapy}

Multiple medical therapies have been described for the treatment of PD $(4,5)$. Medical therapies often recommended for men in the acute phase to stabilize the inflammatory process and to prevent the resultant penile curvature or deformity, for example, men with PD in the acute phase (i.e., $<12$ months in duration) with unstable or progressive deformity, men with established bothersome $\mathrm{PD}$, and patients unwilling to undergo surgical reconstruction (1). Medical therapies include oral medications (vitamin E, potassium aminobenzoate, colchicine, tamoxifen, pentoxifylline and carnitine), intralesional therapy [verapamil, interferon $\alpha-2 b$, and collagenase clostridium histolyticum $(\mathrm{CCH})]$, electromotive, radiation, shockwave therapy and traction (vacuum erection devices). The 2015 American Urological Association (AUA) guidelines on PD details the level of evidence associated with each of these treatment modalities, and discourages the use of oral, electromotive, radiation, and shock wave therapies for the treatment of PD (5). In addition, intralesional injections for the treatment of palpable penile plaque have also been described. In 2013, the Food and Drug Administration (FDA) approved the use of Xiaflex $(\mathrm{CCH})$ for treatment of $\mathrm{PD}$ in patients with palpable plaque and dorsal curvature $\geq 30$ degrees. Its efficacy was demonstrated in two phase III randomized controlled trials [Investigation for Maximal Peyronie's Reduction Efficacy and Safety Studies (IMPRESS I and II)] (6). Prior to $\mathrm{CCH}$, intralesional verapamil and interferon $\alpha-2 b$ have been utilized in the treatment of PD, albeit off-label with varying results.

\section{Therapeutic options for PD: surgical therapy}

Surgical therapies are often recommended for patients with $\mathrm{PD}$ in the chronic established phase with stable plaque and curvature as per AUA guidelines (5). In the absence of $\mathrm{ED}$, there are various surgical therapies available to treat PD such as tunical shortening (plication procedures) and tunical lengthening (incision and grafting). However, when concurrent ED is present, the gold standard treatment is penile prosthetic surgery with or without various straightening procedures. If a clinically significant curvature is persistent after implanting and inflating the cylinders of PP, manual modeling is performed. If a residual curve $>30^{\circ}$ remains after modeling, then additional techniques, including plaque releasing incision, is the next step. Grafting can be considered if tunical defects are $>2.0 \mathrm{~cm}(4,5)$. The current surgical therapies available include tunical shortening procedures such as the Nesbitt and modified plication, tunical lengthening procedures (plaque incision or partial excision and grafting), and PP implantation for men with concurrent $\mathrm{ED}(7)$.

\section{Penile prosthesis and PD}

Men with ED and PD who elect to undergo PP placement deserved special attention and careful surgical planning primarily owing to the associated corporal fibrosis at the level of the plaque, penile shortening and deformity. Detailed patient assessment and counseling are required to ensure realistic patients expectation and to mitigate potential increased complications associated with PD related procedures such as an increased risk of intraoperative crossover, corporal or urethral perforation, or need for a downsized device. Additionally, in cases where adjunctive maneuvers are performed including 
incision or excision of the plaque with or without grafting, the use of cavernotomes, or other specialized dilators and transcorporeal resection. Table 1 summarizes the major published studies discussing the outcomes of surgical treatment of PD with PP.

\section{Patient satisfaction and contemporary outcomes}

Placement of the PP alone is often sufficient to correct penile curvature because the dilatation of the corpora disrupts the fibrotic tissue responsible for the curvature. It is reported that adequate curvature correction after PP placement alone (defined as $<10-20^{\circ}$ ) has been reported in $4-71 \%$ of cases. This wide variation in the reported percentage of adequate straightening with the placement of PP alone among studies are possibly due to the differences in preoperative curvature or extent of dilatation of the corpora at time of PP implantation. Certainly, a more aggressive dilatation, especially with the use of Rossello dilators or cavernotomes, is more likely to break the plaque and corporal fibrosis producing straightening of the penis. Large series have shown that approximately $60-71 \%$ of patients require no additional maneuvers at the time of PP placement, as they have an adequately straight penis already (15-17).

Although malleable and inflatable (PP) both can be used in the setting of $\mathrm{ED}$ and $\mathrm{PD}$, the malleable-type devices are less utilized due to inferior patient satisfaction, higher rates of residual curvature, and less success with subsequent straightening procedures. In one series, dissatisfaction rates of $52 \%$ and $12 \%$ persistent deformity were reported at mean 5 years of follow-up, with dissatisfaction stemming from unnatural erections, cold glans, and narrow penile caliber (14). For the abovementioned reasons, it is generally recommended that an inflatable PP utilized in patients with ED and PD as its cylinders allow for superior expansion with modeling as well as more effective girth enhancement (18).

In regards to selection of the inflatable PP, the American Medical Systems (AMS) CX700 ${ }^{\mathrm{TM}}$ and Titan ${ }^{\mathrm{TM}}$ (Coloplast) are equally effective and considered superior to the AMS Ultrex (AMS) or the AMS LGX ${ }^{\mathrm{TM}}$ (AMS) $(9,19)$. A review by Chung et al. compared the outcomes of 138 patients with ED and PD who underwent placement of either an AMS CX700" ${ }^{\mathrm{TM}}$ (AMS) or Titan $^{\mathrm{TM}}$ (Coloplast) with simultaneous penile modeling (9). In Chung's study, both inflatable PP types provided similar penile straightening without the need for revision surgery, as well as higher patient satisfaction (86\% and $90 \%$, respectively) and improved self-confidence (9). Additionally, the overall 5-year mechanical failure rates between AMS CX700 ${ }^{\mathrm{TM}}$ and Coloplast $\operatorname{Titan}^{\mathrm{TM}}$ were not significantly different (91\% vs. 87\%, P>0.05) (20). When comparing the various AMS 700 cylinders, Montague et al. observed the AMS CX700 ${ }^{\mathrm{TM}}$ to be superior to the AMS 700 Ultrex in PD patients, as it required less corporoplasty maneuvers and caused less postoperative buckling of the inflatable penile prosthesis (IPP) device (15). However, it is still preferable to use the AMS CX700 ${ }^{\mathrm{TM}}$ series over the other AMS devices, such as the AMS Ultrex or AMS 700 LGX, if opting for the use of an AMS product.

\section{Penile modeling technique}

If residual curvature persists intraoperatively after device placement, penile modeling techniques can be employed as an effective and safe option to correct the deformity. These techniques can be offered in patients with dorsal, lateral or dorsolateral curvatures, by a controlled-bending of the penis toward the direction opposite of the maximum curvature and is associated with a risk of urethral rupture. The modeling maneuver as described by Wilson and Delk (18), is performed with a fully inflated prosthesis once the corporotomies have been closed and the exit tubing clamped to prevent pump damage, secondary to back pressure. Protecting the corporotomies with the nondominant hand, the surgeon forcibly bends the penis in the direction opposite the curvature for up to $90 \mathrm{~s}$. The implant is then deflated and reinflated at around $80 \%$ of maximum capacity to allow for re-seating of the implant and to reassess the curvature. If the residual curvature exceeds 20 degrees, the cylinders are inflated to maximum capacity and the modeling procedure is performed a second time if necessary. Inflatable prostheses have shown a clear advantage over malleable devices to correct the residual curvature during the modeling maneuver. Length/girth expansion models (AMS LGX) do not perform as well as girth-only models (AMS CX700 ${ }^{\mathrm{TM}}$ and Coloplast Titan $^{\mathrm{TM}}$ ) during the modeling maneuver $(21,22)$. Levine et al. utilized a surrogate reservoir and rubber shod on the pump tubing to protect the PP from high back pressure, with the goal of gradual curvature correction (20).

In the original Wilson's study, a 3\% urethral perforation rate (18), most likely due to distal extrusion of cylinders at the fossa navicularis (20). Levine et al. placed the bending hand on the shaft rather than on the glans to minimize 
Table 1 A summary of published studies discussing the outcomes of surgical treatment of Peyronie's disease with penile prosthesis

\begin{tabular}{|c|c|c|c|c|c|c|c|c|c|}
\hline $\begin{array}{l}\text { Author/ } \\
\text { year }\end{array}$ & Study & $\mathrm{N}$ & Procedure & $\begin{array}{c}\text { Mean F/U } \\
\text { (mon) }\end{array}$ & $\begin{array}{l}\text { Straightening } \\
(\%)\end{array}$ & $\begin{array}{c}\text { Shortening } \\
(\%)\end{array}$ & $\begin{array}{l}\text { Infection } \\
(\%)\end{array}$ & $\begin{array}{c}\text { Revision/ } \\
\text { removal (\%) }\end{array}$ & $\begin{array}{c}\text { Satisfaction } \\
(\%)\end{array}$ \\
\hline $\begin{array}{l}\text { Egydio, } \\
2013 \text { (8) }\end{array}$ & Prospective & 105 & $\begin{array}{c}\text { IPP + circular \& } \\
\text { longitudinal grafting }\end{array}$ & 18 & 97 & 0 & 1 & 1 & 89 \\
\hline $\begin{array}{l}\text { Chung, } \\
2012(9)\end{array}$ & $\begin{array}{l}\text { Retrospective/ } \\
\text { prospective }\end{array}$ & 138 & $\begin{array}{l}\text { IPP AMS700 CX, } \\
\text { Coloplast Titan }\end{array}$ & 35,40 & 92 & 62 & 2 & 6 & 79 \\
\hline $\begin{array}{l}\text { Silvani, } \\
2012(11)\end{array}$ & Retrospective & 58 & $\begin{array}{l}\text { Soft axial tutors, } \\
\text { single incision \& } \\
\text { saphenous } \\
\text { vein grafting }\end{array}$ & $12-36$ & 100 & 0 & 0 & & 75 \\
\hline $\begin{array}{l}\text { Levine, } \\
2000 \text { (7) }\end{array}$ & Prospective & 46 & IPP & 39 & 100 & 7 & 2 & 0 & NR \\
\hline $\begin{array}{l}\text { Montorsi, } \\
1998(14)\end{array}$ & Prospective & 48 & MPP & 60 & NR & NR & NR & 16 & 48 \\
\hline $\begin{array}{l}\text { Montague, } \\
1996(15)\end{array}$ & Retrospective & 34,38 & $\begin{array}{l}\text { IPP AMS700 CX \& } \\
\text { AMS Ultrex }\end{array}$ & NR & 100,74 & NR & 6,0 & NR & NR \\
\hline
\end{tabular}

IPP, inflatable penile prosthesis; MPP, malleable penile prosthesis; NR, not reported.

downward pressure on the cylinder tips, with the other hand supporting the base of the penis to decrease the chance of suture line disruption (20). Given the force involved, there is some concern for increased rates of inflatable PP failure due to mechanical stress of molding (9).

In a retrospective analysis of 36 patients, Mulhall et al. found that the need for additional intraoperative straightening techniques correlated with the degree of angulation preoperatively (23). They noted that while no maneuvers were absolutely needed for curvatures $\leq 30$ degrees, the need for interventions increased to $12.5 \%$ in curvatures $31-45$ degrees, to $75 \%$ for curvatures $45-60$ degrees, and finally $100 \%$ for curvatures $>60$ degrees (23). With more severe curvatures or curvatures persists greater than 30 degrees after two rounds of manual penile molding, plaque incision, plication or partial excision and grafting may be necessary (24). With tunical defects greater than $2 \mathrm{~cm}$, grafting is recommended in order to decrease the rate of prosthesis herniation (25). Manual modeling might correct the curvature in $61-86 \%$ of patients (18). Success with manual modeling can also depend on the type of device placed, with one study suggesting better outcomes among those receiving inflatable PP $(84 \%$ with corrected curvature) than malleable PP (54\% successfully corrected) $(13,26,27)$.

\section{Additional procedures}

Several studies have evaluated the need for additional procedures such as, corporal plication, plaque incision with or without grafting, to correct penile curvature at the time of PP placement which was found to be in $8-16 \%$ of cases $(19,26,27)$. Wilson has shown that the straightening achieved at the time of device placement is durable, and is not associated with an increased rate of revision for mechanical failure (18). Curvatures of less than 20 degrees do not need to be corrected, as they generally do not interfere with penetrative sexual intercourse and because the regular use of the implant is likely to completely straighten the penis.

Penile lengthening can also be achieved at time of PP placement for patients with ED and PD. In a study of 
23 patients with $\mathrm{PD}$, refractory ED, and severe penile shortening, penile lengthening was performed with a circumferential graft and concomitant placement of an inflatable PP yielding a $2.8 \mathrm{~cm}$ average gain of length (10). For patients and couples who are dissatisfied with penile length postoperatively, Shaeer $e t$ al. have suggested penile elongation and girth augmentation using various flaps, with variable success rates (28). In an initial study by Shaeer et al., an innovative technique of transcorporeal incision of Peyronie's plaques with neither mobilization of the neurovascular bundle nor plaque incision and grafting was reported (12). While Shaeer's results are indeed promising, future studies are prerequisite to further validate its use with patient's satisfaction, safety and outcomes are end-points. Furthermore, Silvani et al. reported a novel technique using a relaxing albugineal incision and saphenous vein grafting in the presence of a soft axially rigid prosthesis cylinder scaffold (11). In Silvani's study, penile elongation enhanced from 1.2 to $2.3 \mathrm{~cm}$ with complete correction of any penile curvature and adequate sexual function (11).

Interestingly, non-operative techniques have also reported to preserve penile length as well as erectile function and penile straightening in the postoperative setting. These include regular penile massage with cocoa butter, penile stretching exercises with traction devices, and nightly use of phosphodiesterase type 5 inhibitors (PDE5i) to promote graft health, with variable results $(19,29)$. More recently, Levine et al. reported their data from a small pilot study using traction therapy before PP placement in men with PD (30). Levine's study demonstrated that following 3-4 months of daily traction for an average of $3 \mathrm{~h}$ or more per day, $70 \%$ of patients gained some length (up to $1.5 \mathrm{~cm}$ ) compared to pre-traction (30).

\section{Postoperative complications}

The main postoperative complaint following inflatable $\mathrm{PP}$ in men with PD is penile shortening. While this has been reported in $50 \%$ of cases, however, it does not impair sexual function (8,31). Egydio et al. performed inflatable PP placement with concomitant circular and longitudinal tunical incisions based on their previously described geometric principles, allowing penile lengthening and girth restoration. In Egydio's study, the overall patient satisfaction rate was $89.4 \%$, but three patients developed graft retraction with residual curves of up to 30 degree and, in one patient, prosthesis explanation was required. Interestingly, there was a mean functional penile length gain of $3.6 \mathrm{~cm}$. Patient satisfaction with penile length gain was $95.2 \%$, and $99 \%$ were able to have satisfactory sexual intercourse (8). Other complications include penile hypoesthesia and paresthesia, infections, difficulties using and deflating the device, mechanical failure, and erosion. Notwithstanding, contemporary published data have shown no significant difference with regards to infection and mechanical failure rates between the $\mathrm{PP}$ and non-PP surgeries performed for PD (10,32-34).

\section{Conclusions}

PD is a debilitating disease that can have a significant impact on one's quality of life. The goal of therapy is to correct the penile deformity to restore sexual function. Penile prosthetic surgery in men with concurrent erectile function have shown to be safe and associated with high patient satisfaction. Various dilation techniques and surgical maneuvers can be performed in conjunction with the placement of PP to achieve a functional result. Despite its promising outcomes, the placement of $\mathrm{PP}$ in a patient with $\mathrm{PD}$ and/or corporal fibrosis can be challenging and is associated with increased morbidities even in the hands of experienced prosthetic surgeons. Therefore, careful patient selection, assessment and counseling should be thoroughly discussed preoperatively to set realistic postoperative expectations for these patients.

\section{Acknowledgements}

None.

\section{Footnote}

Conflicts of Interest: Dr. Hsieh is a consultant for Boston Scientific. The other author has no conflicts of interest to declare.

\section{References}

1. Mulhall JP, Schiff J, Guhring P. An analysis of the natural history of Peyronie's disease. J Urol 2006;175:2115-8.

2. Ralph D, Gonzalez-Cadavid N, MironeV, et al. The management of Peyronie's disease: evidence-based 2010 guidelines. J Sex Med 2010;7:2359-74.

3. Nelson CJ, Mulhall JP. Psychological impact of Peyronie's disease: a review. J Sex Med 2013;10:653-60.

4. Hatzimouratidis K, Eardley I, Giuliano F, et al. EAU 
guidelines on penile curvature. Eur Urol 2012;62:543-52.

5. Nehra A, Alterowitz R, Culkin DJ, et al. Peyronie's Disease: AUA Guideline. J Urol 2015;194:745-53.

6. Gelbard M, Goldstein I, Hellstrom WJ, et al. Clinical efficacy, safety and tolerability of collagenase clostridium histolyticum for the treatment of peyronie disease in 2 large double-blind, randomized, placebo controlled phase 3 studies. J Urol 2013;190:199-207.

7. Levine LA, Dimitrou RJ. A surgical algorithm for penile prosthesis placement in men with erectile failure and Peyronie's disease. Int J Impot Res 2000;12:147-51.

8. Egydio PH, Kuehhas FE, Sansalone S. Penile length and girth restoration in severe Peyronie's disease using circular and longitudinal grafting. BJU Int 2013;111:E213-9.

9. Chung E, Solomon M, DeYoung L, et al. Comparison between AMS 700 ${ }^{\mathrm{TM}} \mathrm{CX}$ and Coloplast ${ }^{\mathrm{TM}}$ Titan inflatable penile prosthesis for Peyronie's disease treatment and remodeling: clinical outcomes and patient satisfaction. J Sex Med 2013;10:2855-60.

10. Sansalone S, Garaffa G, Djinovic R, et al. Simultaneous penile lengthening and penile prosthesis implantation in patients with Peyronie's disease, refractory erectile dysfunction, and severe penile shortening. J Sex Med 2012;9:316-21.

11. Silvani M, Pecoraro S, Zucchi A. Corporoplasty for induratio penis plastica with soft axial tutors, single relaxing albugineal incision and safenous grafting. A 3-year follow up. Arch Ital Urol Androl 2012;84:84-8.

12. Shaeer O. Trans-corporal incision of Peyronie's plaques. J Sex Med 2011;8:589-93.

13. Levine LA, Benson J, Hoover C. Inflatable penile prosthesis placement in men with Peyronie's disease and drug-resistant erectile dysfunction: A single-center study. J Sex Med 2010;7:3775-83.

14. Montorsi F, Guazzoni G, Bergamaschi F, et al. Patientpartner satisfaction with semirigid penile prostheses for Peyronie's disease: A 5-year follow up study. J Urol 1993;150:1819-21.

15. Montague DK, Angermeier KW, Lakin MM, et al. AMS 3-piece inflatable penile prosthesis implantation in men with Peyronie's disease: comparison of CX and Ultrex cylinders. J Urol 1996;156:1633-5.

16. Mulcahy JJ. The Development of Modern Penile Implants. Sex Med Rev 2016;4:177-89.

17. Wilson SK, Cleves MA, Delk JR 2nd. Long-term follow up of treatment for Peyronie's disease: modeling the penis over an inflatable penile prosthesis. J Urol 2001;165:825-9.

18. Wilson SK, Delk 2nd JR. A new treatment for Peyronie's disease: modeling the penis over an inflatable penile prosthesis. J Urol 1994;152:1121-3.

19. Segal RL, Burnett AL. Surgical Management for Peyronie's Disease. World J Mens Health 2013;31:1-11.

20. Levine LA, Larsen SM. Surgery for Peyronie's disease. Asian J Androl 2013;15:27-34.

21. Chung PH, Scott JF, Morey AF. High patient satisfaction of inflatable penile prosthesis insertion with synchronous penile plication for erectile dysfunction and Peyronie's disease. J Sex Med 2014;11:1593-8.

22. Carson CC. Penile prosthesis implantation in the treatment of Peyronie's disease. Int J Impot Res 1998;10:125-8.

23. Mulhall J, Ahmed A, Anderson M. Penile prosthetic surgery for Peyronie's disease: defining the need for intraoperative adjuvant maneuvers. J Sex Med 2004;1:318-21.

24. Austoni E, Colombo F, Romano AL, et al. Soft prosthesis implant and relaxing albugineal incision with saphenous grafting for surgical therapy of Peyronie's disease: a 5-year experience and long-term follow-up on 145 operated patients. Eur Urol 2005;47:223-9.

25. Taylor FL, Abern MR, Levine LA. Predicting erectile dysfunction following surgical correction of Peyronie's disease without inflatable penile prosthesis placement: vascular assessment and preoperative risk factors. J Sex Med 2012;9:296-301.

26. Chaudhary M, Sheikh N, Asterling S, et al. Peyronie's disease with erectile dysfunction: penile modeling over inflatable penile prostheses. Urology 2005;65:760-4.

27. Garaffa G, Minervini A, Christopher NA, et al. The management of residual curvature after penile prosthesis implantation in men with Peyronie's disease. BJU Int 2011;108,1152-6.

28. Shaeer O. Supersizing the penis following penile prosthesis implantation. J Sex Med 2010;7:2608-16.

29. Levine LA, Greenfield JM, Estrada CR. Erectile dysfunction following surgical correction of Peyronie's disease and a pilot study of the use of sildenafil citrate rehabilitation for postoperative erectile dysfunction. J Sex Med 2005;2:241-7.

30. Levine LA, Rybak J. Traction therapy formen with shortened penis prior to penile prosthesis implantation: a pilot study. J Sex Med 2011;8:2112-7.

31. Wang R, Howard GE, Hoang A, et al. Prospective and long-term evaluation of erect penile length obtained with inflatable penile prosthesis to that induced by intracavernosal injection. Asian J Androl 2009;11:411-5. 
32. Carson CC, Levine LA. Outcomes of surgical treatment of Peyronie's disease. BJU Int 2014;113:704-13.

33. Tornehl CK, Carson CC. Surgical alternatives for treating Peyronie's disease. BJU Int 2004;94:774-83.

Cite this article as: Raheem OA, Hsieh TC. Penile prosthetic surgery for the management of Peyronie's disease. Transl Androl Urol 2017;6(Suppl 5):S815-S821. doi: 10.21037/ tau.2017.03.83
34. DiBlasio CJ, Kurta JM, Botta S, et al. Peyronie's disease compromises the durability and component-malfunction rates in patients implanted with an inflatable penile prosthesis. BJU Int 2010;106:691-4. 